\title{
Point Defect Configurations of Supersaturated Au Atoms Inside Si Nanowires
}

2008

Vol. 8, No. 4 1016-1019

\author{
Sang Ho Oh, ${ }^{*, t, \neq}$ Klaus van Benthem,,${ }^{\dagger} \S$ Sergio I. Molina, ${ }^{\dagger, l l}$ Albina Y. Borisevich, ${ }^{\dagger}$ \\ Weidong Luo, ${ }^{\dagger, \#}$ Peter Werner,${ }^{\perp}$ Nikolai D. Zakharov, ${ }^{\perp}$ Dhananjay Kumar, ${ }^{\dagger, \neq}$ \\ Sokrates T. Pantelides, ${ }^{\dagger, \#}$ and Stephen J. Pennycook ${ }^{\dagger, \#}$
}

Materials Science and Technology Division, Oak Ridge National Laboratory,

Oak Ridge, Tennessee 37831, Department of Mechanical and Chemical Engineering,

North Carolina A \& T State University, Greensboro, North Carolina 27411,

Center for Nanophase Materials Sciences, Oak Ridge National Laboratory,

Oak Ridge, Tennessee 37831, Department of Materials Science, Metallurgical

Engineering and Inorganic Chemistry, University of Cadiz, 11510 Puerto Real,

Cadiz, Spain, Max Planck Institute of Microstructure Physics, Weinberg 2,

06120 Halle, Germany, and Department of Physics and Astronomy, Vanderbilt

University, Nashville, Tennessee 37235

Received October 16, 2007; Revised Manuscript Received January 30, 2008

\begin{abstract}
Aberration-corrected scanning transmission electron microscopy (STEM) is used to reveal individual Au atom configurations inside Si nanowires grown by Au-catalyzed vapor-liquid-solid (VLS) molecular beam epitaxy (MBE). We identify a substitutional and three distinct interstitial configurations, one of which has not been previously identified. We confirm the stability of the observed point defect configurations by density functional theory (DFT) calculations. The observed number densities of the various configurations are in accord with their calculated formation energies. The concentration of Au atoms is larger than the solubility limit, but the effect may be caused by the STEM beam.
\end{abstract}

Vertically aligned Si nanowires are commonly grown by the vapor-liquid-solid (VLS) mechanism in which a metal catalyst forms eutectic droplets at the growing tips of the nanowires. ${ }^{1-7}$ A supply of Si vapor supersaturates the droplets with $\mathrm{Si}$ and leads to its precipitation at the liquid-solid (droplet-silicon) interface. Au has been favored as a catalytic material because of the deep eutectic reaction with $\mathrm{Si}$ $\left(\mathrm{Au}_{82} \mathrm{Si}_{18}\right.$ at $\left.360{ }^{\circ} \mathrm{C}\right)$, which enables low temperature growth of Si nanowires $\left(500-650{ }^{\circ} \mathrm{C}\right.$ in most $\left.\operatorname{cases}^{3-7}\right)$. In addition, the low equilibrium solubility (e.g., $2 \times 10^{15} \mathrm{Au}$ atoms $/ \mathrm{cm}^{3}$ in $\mathrm{Si}$ at $\left.650{ }^{\circ} \mathrm{C}\right)^{8}$ and the absence of stable silicides in the $\mathrm{Au}-\mathrm{Si}$ phase diagram suggests growth of clean Si nanowires

* To whom correspondence should be addressed. E-mail: shoh@kbsi.re.kr. Present address: Division of Electron Microscopic Research, Korea Basic Science Institute, Daejeon 305-333, Korea.

$\dagger$ Materials Science and Technology Division, Oak Ridge National Laboratory.

\$ Department of Mechanical and Chemical Engineering, North Carolina A \& T State University.

$\S$ Center for Nanophase Materials Sciences, Oak Ridge National Laboratory.

"Department of Materials Science, Metallurgical Engineering and Inorganic Chemistry, University of Cadiz.

\# Department of Physics and Astronomy, Vanderbilt University.

${ }^{\perp}$ Max Planck Institute of Microstructure Physics. might be possible. However, in situ TEM growth has directly shown that surface migration of Au atoms occurs from the smaller droplets to the larger ones (Ostwald ripening), ${ }^{4}$ suggesting that the sidewalls of Si nanowires are covered by a significant amount of $\mathrm{Au} \cdot{ }^{4,5,7}$ Furthermore, metastable growth techniques such as chemical vapor deposition (CVD) or $\mathrm{MBE}^{9}$ may extend the solubility limit of $\mathrm{Au}$ in $\mathrm{Si}$ nanowires or lead to high nonequilibrium supersaturations. $\mathrm{Au}$ is known to adversely affect the carrier lifetime in $\mathrm{Si}^{8}$ Recent atom probe tomography has indeed demonstrated that $\mathrm{Au}$ catalyst atoms can be incorporated into InAs nanowires beyond the solubility limit. ${ }^{10}$

In this study, we utilize single-atom sensitivity of highangle annular dark field (HAADF) STEM, also known as $Z$-contrast imaging. ${ }^{11,12}$ With the very small depth of focus resulting from the correction of aberrations, ${ }^{13-15}$ imaging and localization of individual $\mathrm{Au}$ atoms in Si nanowires grown by the VLS mechanism using MBE becomes possible. With the three-dimensional resolution, a through-focal series of HAADF images unambiguously distinguishes $\mathrm{Au}$ atoms inside the volume of the nanowire from those decorating the surfaces. Very high number densities of Au atoms are found 


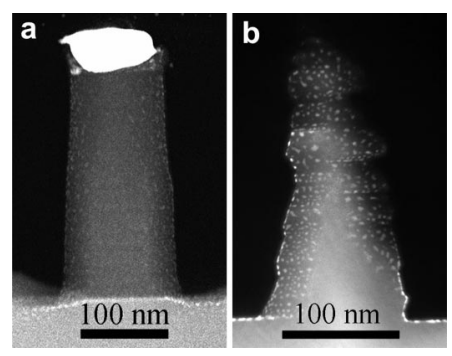

Figure 1. Low magnification HAADF images of Si nanowires. (a) $\mathrm{Si}$ nanowire capped with a solidified $\mathrm{Au}-\mathrm{Si}$ droplet. (b) Faceted and tapered Si nanocone with no solidified droplet. The Z-contrast clearly shows the surface decoration by Au covering the nanowires.

both on the sidewall surfaces and inside the nanowires. Concentrations are estimated in the range of $10^{20} \mathrm{Au}$ atoms/ $\mathrm{cm}^{3}$ closer to the wire surfaces. Although the nonequilibrium nature of the VLS-MBE growth process may contribute to the supersaturation, the STEM beam may be causing the effect through local heating and knock-on events. Furthermore, the projected atomic sites, one substitutional and three distinct interstitial configurations, and their number densities, are directly revealed from the images and agree with the sites and formation energies predicted by DFT calculations. Configurations are seen to change between successive images, consistent with the high diffusivity of $\mathrm{Au}$ in $\mathrm{Si}$, which occurs by interconversion between interstitial and substitutional configurations. ${ }^{16-18}$ Observation at lower temperatures might enable the dynamic observation of diffusion process at the atomic scale.

Vertically aligned Si nanowires were grown on Au-coated Si (111) substrate at $500{ }^{\circ} \mathrm{C}$ by MBE. Details of the growth are described in ref 5. Nanowires were observed in crosssection at ambient temperature with an aberration-corrected STEM operating at $300 \mathrm{kV}$ (VG Microscopes HB603U), an electron probe size of less than $0.08 \mathrm{~nm},{ }^{19}$ and a depth of field of 3-4 nm. ${ }^{14}$ The nanowires were found to be covered by a significant amount of Au nanoclusters, as exhibited by the brighter Z-contrast (Figure 1). Diverse wire morphologies were observed depending on the size of solidified $\mathrm{Au}-\mathrm{Si}$ droplets. The decrease of wire diameter is associated with the reduction in droplet size, which results from the migration of Au. In Figure 1a, for example, it appears that there was a significant reduction in droplet size in the middle of growth. A facetted nanocone in Figure 1b shows no solidified droplet left at the tip, indicating that the surface migration of $\mathrm{Au}$ continued to cause complete depletion of $\mathrm{Au}$ in the droplet. The pronounced surface diffusion may be due to the lowpressure environment of VLS MBE, relative to VLS CVD, where gold surface diffusion would compete with other surface processes. Stacking faults have been observed at the junctions of two different orientations of sidewall facets. ${ }^{20}$

Figure 2 shows three image frames extracted from a HAADF through-focal series recorded near the edge of the Si nanowire. Starting from a far overfocus imaging condition (beam focused before the specimen), a total number of 41 frames were acquired successively with focus advancing into the sample in $2 \mathrm{~nm}$ steps. The through-focal imaging
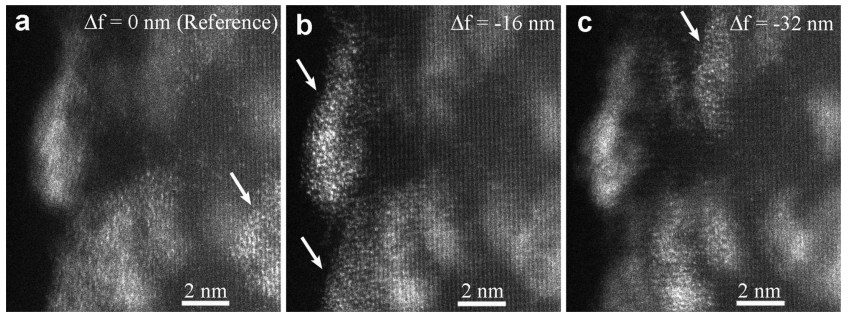

Figure 2. Three frames selected from a through-focal series of HAADF images acquired in $2 \mathrm{~nm}$ defocus increments in the crosssectional $\langle 112\rangle$ orientation. Au (single atoms and nanoclusters) atoms existing at different surface locations can be identified. In each frame, the Au nanoclusters in focus are indicated by arrows: (a) on the top sidewall at $0 \mathrm{~nm}$, (b) at the edge sidewall at -16 $\mathrm{nm}$, and (c) on the bottom sidewall at $-32 \mathrm{~nm}$ relative defocus.
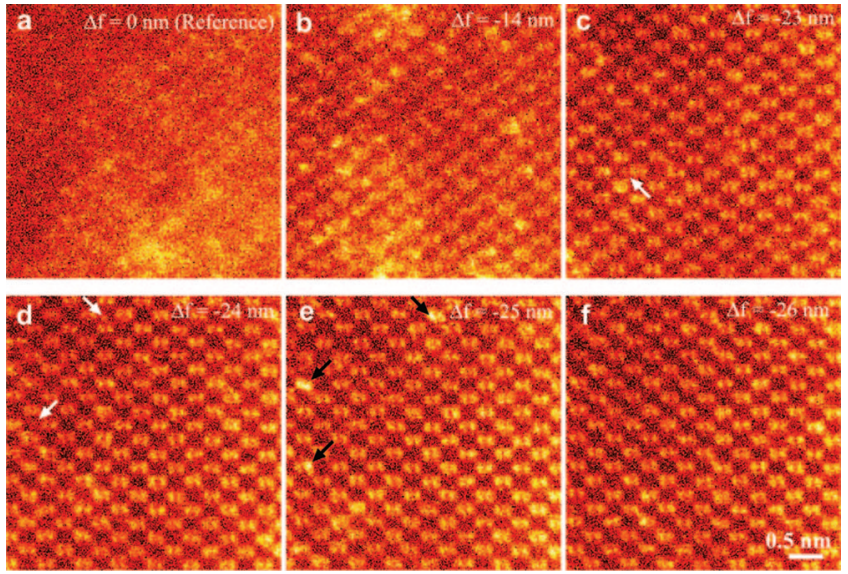

Figure 3. Six frames selected from a through-focal series of HAADF images acquired with $1 \mathrm{~nm}$ defocus steps at the crosssectional $\langle 110\rangle$ orientation. The in-focus plane is located (a) above and (b) near the top surface of the nanowire. Four successive frames were acquired when the electron beam is focused in the center of the nanowire volume at relative defocus values of (c) $-23 \mathrm{~nm}$, (d) $-24 \mathrm{~nm}$, (e) $-25 \mathrm{~nm}$, (f) $-26 \mathrm{~nm}$.

technique and the depth resolution are treated in more detail in ref 14 . When the top surface of the thick region of the nanowire comes into focus along with surface Au atoms (the right-hand side of Figure 2), the corresponding defocus value is set as reference (Figure 2a). Further decrease of defocus by $16 \mathrm{~nm}$ brings the edge of the nanowire into focus (Figure $2 \mathrm{~b}$ ), while the Au atoms at the bottom surface come into focus only after decreasing the defocus by an additional 16 $\mathrm{nm}$ (Figure 2c). Therefore, in the thick part of the nanowire in the center of Figure $2 b$, the beam is focused inside the nanowire, when atoms on the top and bottom surfaces are not in focus and are not visible in the image.

HAADF through-focal series were then acquired at higher magnifications with finer defocus increments of $1 \mathrm{~nm}$ and revealed $\mathrm{Au}$ atoms to be present inside the $\mathrm{Si}$ nanowires at both interstitial and substitutional sites. Figure 3 shows six frames extracted from such a through-focal series. When the electron beam is focused near the top surface region, the surface $\mathrm{Au}$ atoms and/or the substitutional atoms located just below the surface are in focus (Figure 3b). As the electron probe is moved toward the center of the nanowire, interstitial $\mathrm{Au}$ atoms appeared while the number of substitutional $\mathrm{Au}$ 


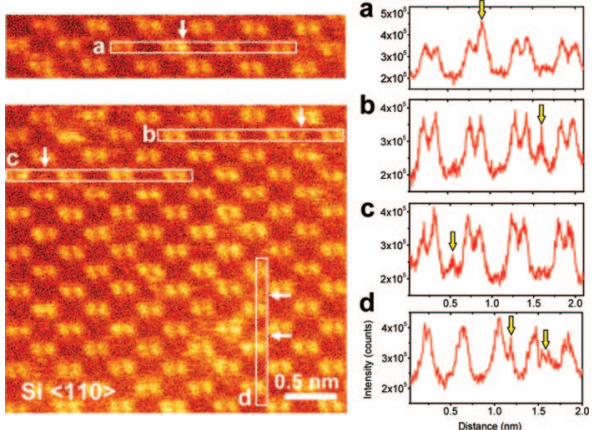

Figure 4. HAADF images of a $\mathrm{Si}$ nanowire in $\langle 110\rangle$ zone-axis orientation (left panel). A slight image distortion caused during the scan was unwarped. Boxes show the regions used for intensity profiles, with $\mathrm{Au}$ atoms in various configurations arrowed: (a) substitutional, (b) tetrahedral, (c) hexagonal, (d) buckled $\mathrm{Si}-\mathrm{Au}-\mathrm{Si}$ chain configurations. The intensity profiles across the Si dumbbells correspond to a width of 18 pixels.

atoms decreased significantly. Parts $\mathrm{c}-\mathrm{f}$ in Figure 3 show four successive frames, where some interstitial Au atoms are indicated by white arrows. Far fewer interstitial Au atoms are observed than substitutional Au atoms (some of which are marked by black arrows in Figure 3e). It should be noted that the Z-contrast features we attribute to Au atoms are not seen in images of pure $\mathrm{Si}$ samples or of the substrate on which the nanowires are grown. Finally, it is noted that the positions of $\mathrm{Au}$ atoms change from frame to frame, indicating that they are migrating, either thermally or assisted by electron beam heating or knock-on displacement. Even repeating scans over the same area with a fixed defocus do not find the same $\mathrm{Au}$ atoms in the same configurations. In a qualitative way, therefore, one caninferthatsubstitutional-interstitial conversions occur, in accord with the known mechanism of $\mathrm{Au}$ diffusion in $\mathrm{Si}^{16}$

Close inspection of HAADF images reveals interstitial Au atoms located not only at the empty tetrahedral sites in the Si lattice but also in other configurations. There are four empty tetrahedral sites available in the unit cell of Si while the other four are regular atomic sites. Note that the atomic packing factor of the diamond structure of $\mathrm{Si}$ is only 0.34 , providing a large open space for the accommodation of interstitial defects. Figure 4 shows HAADF images from the center of the nanowire, where individual $\mathrm{Au}$ atoms are marked by arrows. Accompanying line scans clearly show the extra intensity introduced by $\mathrm{Au}$ atoms to be statistically significant. Along with the substitutional configuration (Figure 4a), three interstitial configurations are observed, which are: tetrahedral (Figure 4b), hexagonal (Figure 4c), and a buckled $\mathrm{Si}-\mathrm{Au}-\mathrm{Si}$ chain (Figure 4d). The hexagonal site appears in the middle of two neighboring tetrahedral sites, at which the Au atoms are surrounded by six neighboring $\mathrm{Si}$ atoms. The third configuration was observed less frequently, with the interstitial Au atom located in a buckled $\mathrm{Si}-\mathrm{Au}-\mathrm{Si}$ configuration. A substitutional $\mathrm{Au}$ atom located in one of the $\mathrm{Si}$ (400) dumbbell columns shows an intensity rise by $\sim 30 \%$ compared to the average intensity of $\mathrm{Si}$ columns. The HAADF intensity scattered by a single interstitial $\mathrm{Au}$ atom is comparable to $\sim 50 \%$ of the intensity

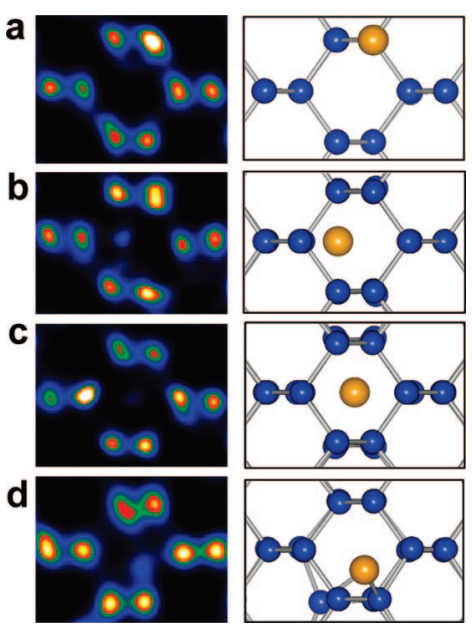

Figure 5. Local views of defect configurations of $\mathrm{Au}$ in $\mathrm{Si}$. (a) Substitutional, (b) tetrahedral, (c) hexagonal, (d) buckled $\mathrm{Si}-\mathrm{Au}-\mathrm{Si}$ chain configurations. The HAADF images (left panel) were processed by maximum entropy image deconvolution (HREM Research Inc.) using a Lorentz-type probe function. The corresponding atomic models (right panel) calculated by DFT match the images closely.

of the neighboring Si columns. These intensity levels and the range of focus are consistent with image simulations for single substitutional and interstitial Au atoms (see Supporting Information).

Stable configurations of isolated Au impurities in Si were determined using DFT. The point defects are simulated based on a cubic supercell containing $64 \mathrm{Si}$ atoms with an additional $\mathrm{Au}$ atom at a particular configuration. DFT calculations in the generalized-gradient approximation (GGA) were performed using the Vienna $\mathrm{Ab}$ Initio Simulation Package (VASP) code and projector-augmented-wave (PAW) method. ${ }^{21,22}$ Bulk Si is assumed to define the Si chemical potential. The energetically most favorable configuration for a single $\mathrm{Au}$ atom inside the Si lattice was found to be the substitutional site. This is consistent with both our experimental atomic-scale observations and with earlier diffusion studies, ${ }^{18}$ which show that $\mathrm{Au}$ atoms in $\mathrm{Si}$ are predominantly substitutional defects. The formation energy of a substitutional $\mathrm{Au}$ atom is taken as a reference, to which the formation energies for interstitial configurations are compared. Two common interstitial defect configurations, with single $\mathrm{Au}$ atoms positioned at the tetrahedral and the hexagonal-ring interstices surrounded by $\mathrm{Si}$ atoms, had relative formation energies of 1.33 and $1.46 \mathrm{eV}$, respectively. For the new defect configuration, Figure $4 d$, the stable configuration was found to consist of a $\mathrm{Au}$ atom inserted into a $\mathrm{Si}-\mathrm{Si}$ bond, forming a buckled $\mathrm{Si}-\mathrm{Au}-\mathrm{Si}$ bond (bond angle $108^{\circ}$ ). This configuration also has a comparable relative formation energy of $1.68 \mathrm{eV}$, with the $\mathrm{Au}$ interstitial located about $1.4 \AA$ from the center of the undeformed $\mathrm{Si}-\mathrm{Si}$ bond and 2.36 and 2.40 $\AA$ from its Si neighbors. This configuration corresponds to the observed defect in Figure 4d. Magnified images after maximum entropy processing compare very well to the DFT structures, see Figure 5.

The relative number density of each interstitial configuration was estimated by direct count from several large-area 
Table 1. Summary of the Defect Formation Energies Calculated by DFT $\left(E_{\mathrm{i}}-E_{\mathrm{s}}\right)$ and the Relative Number Densities of Each Interstitial Configuration Determined by Direct Count from HAADF Images $\left(N_{\mathrm{i}} / N_{\mathrm{s}}\right)$.

\begin{tabular}{lcc}
\hline \multicolumn{1}{c}{$\begin{array}{c}\text { interstitial } \\
\text { configuration }\end{array}$} & $E_{\mathrm{i}}-E_{\mathrm{s}}(\mathrm{eV})$ & $N_{\mathrm{i}} / N_{\mathrm{s}}$ \\
\hline tetrahedral & 1.33 & $0.12 \pm 0.05$ \\
hexagonal & 1.46 & $0.08 \pm 0.03$ \\
buckled $\mathrm{Si}-\mathrm{Au}-\mathrm{Si}$ & 1.68 & $0.05 \pm 0.04$
\end{tabular}

HAADF images and different defocus levels. A larger number of substitutional Au atoms is counted closer to the wire surface, in agreement with the typical diffusion profile that $\mathrm{Au}$ attains in $\mathrm{Si}$, the so-called "U-shape" profile. ${ }^{18}$ The count of each interstitial configuration was divided by the total number of substitutional atoms counted in the corresponding image. Then, these relative numbers obtained from different images were averaged. The resulting relative number densities follow the ordering that would be predicted from the calculated formation energies, i.e., lower formation energy implying higher density (Table 1 ). We stress that number densities are only approximate due to a different depth of focus for interstitial and substitutional atoms and possible double counting as a result of atom migration.

The relative defect densities decrease as the formation energy increases, but fitting an Arrhenius-type equation, the local temperature is estimated in excess of $1000{ }^{\circ} \mathrm{C}$. A possible interpretation of this result is that the beam causes interconversion among interstitial configurations both by momentum transfer and local heating. A similar phenomenon has been demonstrated to occur in Si grain boundaries, where beam-induced vacancy creation initially occurs selectively in columns with low vacancy formation energies. ${ }^{23}$

In summary, we report observations of catalyst $\mathrm{Au}$ atoms inside Si nanowires. Four different Au point defect configurations are distinguished in $Z$-contrast images, which were confirmed through multislice image simulations and DFT calculations. The observed number densities of the various configurations are in accord with their calculated formation energies. The present work demonstrates that direct imaging of distinct impurity configurations in a crystal and determination of relative densities is feasible by aberration-corrected STEM. These results, perhaps in conjunction with detailed simulations of beam-crystal interactions, set the stage for the extraction of information about point defect dynamics and defect reactions in nanostructures.

Acknowledgment. This work was supported by the Office of Basic Energy Sciences, Divisions of Materials Sciences and Engineering (A.Y.B., S.J.P.) and Scientific User Facilities (A.Y.B., K.v.B.), an NSF-NIRT project (DMR-0403480, S.H.O., D.K.), the NODE and SANDiE European Networks of Excellence (contract no. NMP4-CT-2004-500101), the SpanishMEC (TEC2005-05781-C03-02) and the Junta de Andalucia (PAI research group TEP-120; project PAI05TEP-383, S.I.M.), the European Union (NODE, FP6 /015783, P.W., N.D.Z.), and the McMinn Endowment at Vanderbilt University (STP).

Supporting Information Available: Multislice simulation of STEM HAADF images. This material is available free of charge via the Internet at http://pubs.acs.org.

\section{References}

(1) Levitt, A. P. Whisker Technology; John Wiley \& Sons: New York, 1970.

(2) Givargizov, E. I. J. Cryst. Growth 1975, 31, 20.

(3) Ross, F. M.; Tersoff, J.; Reuter, M. C. Phys. Rev. Lett. 2005, 95, 146104.

(4) Hannon, J. B.; Kodambaka, S.; Ross, F. M.; Tromp, R. M. Nature 2006, 440, 69.

(5) Werner, P.; Zakharov, N. D.; Gerth, G.; Schubert, L.; Gösele, U. Int. J. Mater. Res. 2006, 97, 1007.

(6) Kodambaka, S.; Hannon, J. B.; Tromp, R. M.; Ross, F. M. Nano Lett. 2006, 6, 1292.

(7) Cao, L.; Garipcan, B.; Atchison, J. S.; Ni, C.; Nabet, B.; Spanier, J. E. Nano Lett. 2006, 6, 1852.

(8) Collins, C. B.; Carlson, R. O.; Gallagher, C. J. Phys. Rev. 1957, 105, 1168.

(9) Williams, J. S.; Short, K. T. J. Appl. Phys. 1982, 53, 8663.

(10) Perea, D. E.; Allen, J. E.; May, S. J.; Wessels, B. W.; Seidman, D. N.; Lauhon, L. J. Nano Lett. 2006, 6, 181.

(11) Voyles, P. M.; Muller, D. A.; Grazul, J. L.; Citrin, P. H.; Gossmann, H.-J. L. Nature 2002, 416, 826.

(12) Lupini, A. R.; Pennycook, S. J. Ultramicroscopy 2003, 96, 313.

(13) van Benthem, K.; Lupini, A. R.; Kim, M.; Baik, H. S.; Doh, S. J.; Lee, J.-H.; Oxley, M. P.; Findlay, S. D.; Allen, L. J.; Pennycook, S. J. Appl. Phys. Lett. 2005, 87, 034104.

(14) van Benthem, K.; Lupini, A. R.; Oxley, M. P.; Findlay, S. D.; Allen, L. J.; Pennycook, S. J. Ultramicroscopy 2006, 106, 1062.

(15) Borisevich, A. Y.; Lupini, A. R.; Pennycook, S. J. Proc. Natl. Acad. Sci. U.S.A. 2006, 103, 3044.

(16) Gösele, U.; Frank, W.; Seeger, A. Appl. Phys. 1980, 23, 361.

(17) Nichols, C. S.; Van de Walle, C. G.; Pantelides, S. T. Phys. Rev. Lett. 1989, 62, 1049.

(18) Murch, G. E.; Nowick, A. S. Diffusion in Crystalline Solids; Academic Press Inc.: Orlando, 1984.

(19) Nellist, P. D.; Chisholm, M. F.; Dellby, N.; Krivanek, O. L.; Murfitt, M. F.; Szilagyi, Z. S.; Lupini, A. R.; Borisevich, A.; Side, W. H., Jr.; Pennycook, S. J. Science 2004, 305, 1741.

(20) Hao, Y.; Meng, G.; Wang, Z. L.; Ye, C.; Zhang, L. Nano Lett. 2006, 6, 1650.

(21) Kresse, G.; Furthmuller, J. Phys. Rev. B 2006, 54, 11169.

(22) Blochl, P. E. Phys. Rev. B 1994, 50, 17953.

(23) Maiti, A.; Pantelides, S. T.; Chisholm, M. F.; Pennycook, S. J. Appl. Phys. Lett. 1999, 75, 2380.

NL072670+ 